\title{
A PROPERTY-SHARING CONSTRAINT IN CENTERING
}

\author{
Megumi Kameyama \\ Department of Computer and Information Science \\ The Moore School of Electrical Engineering/D2 \\ University of Pennsylvania \\ Philadelphia, PA 19104
}

\begin{abstract}
${ }^{1}$
A constraint is proposed in the Centering approach to pronoun resolution in discourse. This "property-sharing" constraint requires that two pronominal expressions that retain the same $\mathrm{Cb}$ across adjacent utterances share a certain common grammatical property. This property is expressed along the dimension of the grammatical function SUBJECT for both Japanese and English discourses, where different pronominal forms are primarily used to realize the $\mathrm{Cb}$. It is the zero pronominal in Japanese, and the (unstressed) overt pronoun in English. The resulting constraint complements the original Centering, accounting for its apparent violations and providing a solution to the interpretation of multi-pronominal utterances. It also provides an alternative account of anaphora interpretation that appears to be due to structural parallelism. This reconciliation of centering/focusing and parallelism is a major advantage. I will then add another dimension called the "speaker identification" to the constraint to handle a group of special cases in Japanese discourse. It indicates a close association between centering and the speaker's viewpoint, and sheds light on what underlies the effect of perception reports on pronoun resolution in general. These results, by drawing on facts in two very different languages, demonstrate the cross-linguistic applicability of the centering framework.
\end{abstract}

\section{Introduction}

Grosz, Joshi, \& Weinstein (1983) postulated that each utterance in discourse concerns a set of entities called the centers, and discussed how certain facts of local discourse connectedness (as opposed to global) can be accounted for

\footnotetext{
'This work was supported in parts by the Center for the Study of Language and Information at Stanford University and by grants from the National Science Foundation (DCR84-11726) for the Department of Computer and Information Science and from the Alfred P. Sloan Foundation for the Cognitive Science Program at the University of Pennsylvania.
}

using this notion. ${ }^{2}$

Centers are semantic objects--(sets of) individuals, objects, states, actions, or events--represented in complex ways so that a strict coreference need not hold between anaphorically related terms. ${ }^{3}$ A center mentioned in the current utterance may be mentioned again in the next utterance (by the same or a different speaker). In this sense, a center is "forward-looking" (CI). Crucially, one of the centers may be identified as "backward-looking" (Cb). $\mathrm{Cb}$ is the entity an utterance most centrally concerns. Its main role is to connect the current utterance to the preceding one(s). ${ }^{4}$ The term the Center is also used for the $\mathrm{Cb}$. Thus an utterance may be associated with any number of $\mathrm{Cfs}$, one of which may be the $\mathrm{Cb}$. These $\mathrm{Cfs}$ are given a default expected $\mathrm{Cb}$ order, that is, "how much each center is expected to be the next $\mathrm{Cb}^{\prime}$. I regard $\mathrm{Cb}$ to be optional for an utterance. 5 It comes into exsistence by way of a Cb-establishment process, that is, the process in which a previous non- $\mathrm{Cb}$ becomes the new $\mathrm{Cb}$ in discourse. Sidner's $(1981,1983)$ immediate focus and potential foci in local focusing correspond to $\mathrm{Cb}$ and $\mathrm{Cfs}$, respectively. The difference is that Sidner uses two immediate foci (Discourse Focus and Actor Focus) while centering uses only one (Cb) (see Grosz et. al. 1983 for discussion).

Various factors --syntactic, semantic, and pragmatic-are combined for the identification of the $\mathrm{Cb}$. One of them is the use of pronominal expressions, as expressed in the

\footnotetext{
${ }^{2}$ In a theory of discourse structure that consists of three interacting components, linguistic, intentional, and attentional (Grosz \& Sidner 1985), centers are found in the local attentional structure.

${ }^{3}$ See Sidner's (1979) focus representation for instance.

4The notion of centering originally comes from Joshi \& Weinstein (1981).

${ }^{5}$ We can view $\mathrm{Cb}$ either optional or obligatory for each utterance. The difference seems more conceptual than substantial since what is crucial for providing a referent candidate is the expected $\mathrm{Cb}$ order given to the $\mathrm{Cf}$ set whether this set contains the $\mathrm{Cb}$ or not. Relative merits of each approach should be clarified in the future.
} 
original Centering rule (Grosz et. al. 1983): ${ }^{6}$

(1) If the $\mathrm{Cb}$ of the current utterance is the same as the $\mathrm{Cb}$ of the previous utterance, a pronoun should be used.

(1) is stated as a heuristic in the production of English. It is assumed that an equivalent interpretation heuristic is used by a hearer. Roughly, a pronoun "realizes" the current $\mathrm{Cb}$ that continues the previous $\mathrm{Cb}^{\text {? }}$ ?

In this paper, I will first point out certain facts that the basic Centering rule does not explain, then propose a further constraint that substantiates the basic rule. This is called the "property-sharing" constraint, which requires that two pronominal elements realizing the same $\mathbf{C b}$ in adjacent utterances share a certain common grammatical property. This shared property itself is expressed as a default preference order reflecting the nature of the constraint as a discourse rule. The initial formulation of the constraint only refers to the grammatical function SUBJECT. It explains the problem cases for the basic Centering rule in Japanese and English. It also accounts for a subset of what appears to be an effect of structural parallelism in anaphora interpretation. Then I will propose an additional dimension of the shared property called the "speaker identification" property. The revised constraint referring to both dimensions accounts for a group of counterexamples to the initial formulation found in Japanese discourse. It also sheds light on what is involved in interpreting perception reports in both languages.

Before starting the discussion, I would like to comment on the nature of the data used here. I will mostly use constructed discourse sequences where the role played by commonsense inferences or special linguistic devices (such as stress and intonation) for guiding pronoun interpretations is minimal. All examples in this paper are to be read with flat intonation with unstressed pronouns. These limitations are in order to identify the grammatically-based default order that gives rise to preferred interpretations in neutral contexts. Note that this default order alone does not determine interpretations of pronominal elements. Rather, its role in the centering framework is to give an ordered list of referents (centers) so that commonsense inferences can be controlled. Interpretations and acceptability judgements of the examples in this paper result from interviews with a number of native speakers in each language.

\footnotetext{
${ }^{6}$ Grosz et. al. (in preparation) propose various constraints on this rule, and, among other things, distinguish between the retention and continuation of the $\mathrm{Cb}$. I will use the words retain and continue in non-technical sense in this paper.

${ }^{7}$ An expression realizes a center rather than denoting it. Realization allows either a value-free or value-loaded interpretation (see Grosz et. al. 1983 for discussion).
}

\section{The SUBJECT constraint}

\subsection{Japanese}

In Japanese, the expression primarily used to realize the $\mathrm{Cb}$ is the zero pronominal (i.e., unexpressed subject or object). 8 The grammatical features (e.g., gender, number, person) of these unexpressed subjects and objects are not morphologically marked elsewhere in the sentence, which distinguishes them from the so-called "pro-drop", such as the unexpressed finite clause subject in Italian and Spanish whose grammatical features are morphologically marked on the verb inflection. The basic Centering rule in Japanese can be obtained by changing the word pronoun to zero pronominal in (1) (Kameyama 1985).

In the following discourse fragment, it is reasonable to assume that Rosa is the $\mathrm{Cb}$ of the second utterance: ${ }^{9}$

(2)

1. Rosa wa dare o matte-iru no-desu ka.

Rosa TP-SB who OB is-waiting-for ASN $Q$

"Who is Rosa waiting for?"

2. Ф Mary o matte-iru no-desu.

SB Mary OB is-waiting-for ASN

"[She] is waiting for Mary." [Cb=Rosa]

It seems equally reasonable to assume that Rosa is the $\mathrm{Cb}$ of the second utterance in the following variation of (2):

(3)

1. Dare ga Rosa o matte-iru no-desu ka. who SB Rosa OB is-waiting-for ASN Q "Who is waiting for Rosa?"

2. Mary ga $\Phi$ matte-iru no-desu. Mary SB OB is-waiting-for ASN

"Mary is waiting for [her]." [Cb=Rosa]

If the Cb-status of an entity is homogeneous, we would expect that the two instances of the $\mathrm{Cb}$ above have exactly the same effect, if any, on the subsequent utterance. When an identical third utterance is added to both, however, it becomes clear that the centered individual Rosa is not of an equal status in the two cases:

\footnotetext{
${ }^{8}$ Zero pronominals are also found in Chinese, Korean, Vietnamese, Thai, etc. I will also call them "zero-subject", "zero-object", and so on.

${ }^{9}$ The following symbols are used for grammatical markers in the gloss: SB (subject), OB (direct object), O2 (indirect/ second object), TP (topic), ASN (assertion), CMP (complementizer), $Q$ (question). The symbol $\Phi$ is used for a zero pronominal, and its translation equivalent appears in [].
} 
(4)

$\Phi$ Ф Yuusyoku ni syootaisi-ta no-desu.

SB OB supper to invited ASN

"[She] invited [her] to dinner."

after(2): [strong preference: Rosa invited Mary]

after(3): [weak preference: Mary invited Rosa]

(5)

Ф Rosa ni yuusyoku ni syootais-are-ta no-desu.

SB Rosa by supper to was-invited ASN

"[She] was invited by Rosa to dinner." (she $=:$ Mary) ${ }^{10}$

after(2): marginal (*?)

after(3): acceptable

The extension (4) is a multi-zero-pronominal utterance. The zero-subject and zero-object pronominals receive reverse interpretations depending on whether the utterance follows (2) or (3). Although this fact by itself does not contradict the basic rule (1), it poses a question as to which zero pronominal in (4) realizes its $\mathrm{Cb}$. There are the following two possibilities. If the previous $\mathrm{Cb}$ continues to be the current $\mathrm{Cb}$ by default, it follows that the choice of the $\mathrm{Cb}$-realizing zero pronominal depends entirely on the preceding discourse context. On the other hand, if some inherent property of a zero pronominal (e.g., subject/object) independently decides which one realizes the $\mathrm{Cb}$, the previous context need not be considered. For instance, if a zero-subject is always more closely associated with the $\mathrm{Cb}$ than a zero-object, the discourse sequence (3) to (4) changes the $\mathrm{Cb}$ from Rosa to Mary.

In the extension (5), Rosa (the previous $\mathrm{Cb}$ ) is mentioned with a full name while the single zero pronominal picks out a previous non- $\mathrm{Cb}$, Mary. If Rosa is still the $\mathrm{Cb}$ here, this utterance violates the basic Centering rule, so the rule predicts unacceptability, which is indeed the case following the sequence (2). 11 The same rule, however, provides no clue for the puzzling acceptability of the same extension following the sequence (3). Moreover, it is possible that Rosa is no longer the $\mathrm{Cb}$ in (5), in which case, rule (1) simply does not apply.

Examples like these are the basis for the first version of the Centering Constraint:

(6) Centering Constraint [Japanese] (1st approximation) Two zero pronominals that retain the same $\mathrm{Cb}$ in adjacent utterances should share one of the following properties:

\footnotetext{
${ }^{10}=$ : indicates the association between a linguistic item (left-hand side) and a non-linguistic entity (right-hand side).

${ }^{11}$ Note that violating a discourse rule like (1) leads to more difficulty in understanding rather than clear-cut "ungrammaticality".
}

\section{SUBJECT or nonSUBJECT..$^{12}$}

(6) says that two zero pronominals supporting the same $\mathrm{Cb}$ in adjacent utterances should both be either SUBJECT or nonSUBJECT. In the case of discourse extension (4) above, if the $\mathrm{Cb}$ is still Rosa, it should be realized with a zero-subject after the sequence (2) and with a zero-object after (3). This is shown below:

(7)
1. $[\mathrm{Cb}<\mathrm{SUBJ}>=\mathrm{Rosa}]<--(2)-2$
2. [Cb $<S U B J>=R o s a]<--(4)$ [strong preference]

(8)

1. $[\mathrm{Cb}<\mathrm{OBJ}>=\mathrm{Rosa}]<-(3)-2$

2. $[\mathrm{Cb}<\mathrm{OBJ}>=\mathrm{Rosa}]<-(4)$ [weak preference]

I attribute the different degree of preference between (7) and (8) to the difference in canonicality of centering. $\mathrm{A} \mathrm{Cb}$ continued with zero-subjects as in (7) is more stable, or more canonical, than one continued with zero-objects as in (8), which is but one manifestation of the overall significance of SUBJECT in centering. ${ }^{13}$ This leads to the second approximation of the Centering Constraint:

(9) Centering Constraint [Japanese] (2nd
approximation) Two zero pronominals that retain the same $\mathrm{Cb}$ in adjacent utterances should share one of the following properties (in descending order of preference): 1) SUBJECT, 2) nOnSUBJECT.

Constraint (9) predicts that retaining $\mathrm{a} \mathrm{Cb}$ is good when the two pronominals are both either SUBJECT or nonSUBJECT while it is bad (i.e., leading to complex inferences) when one is SUBJECT and the other is not. This in tum predicts that changing the $\mathrm{Cb}$ across adjacent utterances is acceptable when the two pronominals have different properties while it is not when they are of the same property.

The difference in acceptability between sequence (2) to (5) (marginal) and sequence (3) to (5) (acceptable) would then follow from this constraint. The former is bad because it changes the $\mathrm{Cb}$ with two SUBJECT zero pronominals, as shown in (10). The latter is good because it changes the $\mathrm{Cb}$ with different zero pronominals (from OBJECT to SUBJECT), as shown in (11):

$$
\begin{aligned}
& \text { (10) } \\
& \text { 1. }[\mathrm{Cb}<\mathrm{SUBJ}>=\mathrm{Rosa}]<-(2)-2 \\
& \text { 2. *? [Cb<SUBJ> = Mary] <--(5) [marginal] }
\end{aligned}
$$

\footnotetext{
12 'm referring to the "surface" grammatical function SUBJECT.

${ }^{13}$ The importance of SUBJECT in centering is also discussed in Grosz et. al. (in preparation).
} 
The acceptability of the Cb-shift shown in (11) above contrasts with the unacceptability of retaining the $\mathrm{Cb}$ with these pronominals. The latter in fact appeared in the above example as the nonpreferred reading of sequence (3) to (4), which is shown in (12):

(12)

1. $[\mathrm{Cb}<\mathrm{OBJ}>=\mathrm{Rosa}]$

2. ?? [Cb $<$ SUBJ $>=$ Rosa $]$

\subsection{English}

The following sequences in English are equivalent to those in Japanese (2) to (5):

(13)

1. Who is Max waiting for?

2. He is waiting for Fred. $[\mathrm{Cb}<\mathrm{SUBJ}>=\mathrm{Max}]$

3a. He invited him to dinner. [strong preference: Max invited Fred]

3b. ?* He was invited by Max to dinner.

(14)

1. Who is waiting for Max?

2. Fred is waiting for him. $[\mathrm{Cb}<$ nonSUBJ $>=\mathrm{Max}]$

3a. He invited him to dinner.

[weak preference: Fred invited Max]

3b. (?) He was invited by Max to dinner.

The evaluation of the third utterance parallels the Japanese example. This indicates that the SUBJECT-based constraint stated in (9) for Japanese is applicable to English together with all the analogous consequences discussed above. The constraint is restated below for pronominal expressions in general:

(15) Centering Constraint [general] (approximation) Two pronominal expressions that retain the same $\mathrm{Cb}$ in adjacent utterances should share one of the following properties (in descending order of preference): 1) SUBJECT, 2) nonSUBJECT.

The particular kind of pronominal expressions relevant here vary from language to language. Kameyama (1985: Ch.1) hypothesized that it is the pronominal element with the "less phonetic content" for each grammatical function of a language ${ }^{14}$ and that it is predictable from the typological perspective on available pronominal forms. For instance, it is the unstressed pronoun in English where pronouns must always be overt, and it is the zero pronominal in Japanese where pronouns with no phonetic

\footnotetext{
${ }^{14}$ It is possible that only certain grammatical functions (e.g., SUBJECT, OBJECT, and OBJECT2) are relavant to the $\mathrm{Cb}$. This will have to be clarified in the future.
}

content exist (for subjects and objects). It is further predicted that morphologically bound pronominal forms (i.e., agreement inflections, clitics, and affixes) rather than full independent pronouns are used for $\mathrm{Cb}$-realization if a language has this option. For instance, this option exists for the finite clause subject in Italian and Spanish in terms of the agreement inflection, and for the finite clause subject and object in Warlpiri in terms of clitics. The constraint in English is stated below:

(16) Centering Constraint [English]

Two unstressed pronouns that retain the same $\mathrm{Cb}$ in adjacent utterances should share one of the following properties (in descending order of preference): I) $S U B J E C T, 2)$ nonSUBJECT.

\subsection{Accounting for the effect of parallelism in Cb-establishment}

The given property-sharing constraint has so far been proposed for pronominal elements that retain the same $\mathbf{C b}$ in adjacent utterances. By its reference to the grammatical property SUBJECT, the constraint indicates that adjacent utterances of the same $\mathrm{Cb}$ cohere even better when there is a certain degree of grammatical parallelism.

Analogous constraints account for at least two other kinds of parallelism effects on pronoun interpretation in English. They are in the context of what I call the Cb-establishment, that is, the process in which a previous non- $\mathrm{Cb}$ becomes the $\mathrm{Cb}$. The case of $\mathrm{Cb}$-shift is a subcase of $\mathrm{Cb}$-establishment. ${ }^{15}$

Ambiguous multl-pronouns. The first is the interpretation of a multi-pronominal utterance that establishes a $\mathrm{Cb}$. An example follows:

(17)

1. Max is waiting for Fred.

2. He invited him to dinner. [preference: Max invited Fred]

(17) shows that when two pronouns are potentially ambiguous in reference, the preferred interpretation conforms to a property-sharing constraint. That is, there is a higher tendency that the SUBJECT pronoun corefers with the SUBJECT of the previous utterance.

It is crucial here that (a) there is more than one pronoun and (b) two (or more) of them are potentially ambiguous (i.e., of the same grammatical features). Otherwise, the process of $\mathrm{Cb}$-establishment need not be constrained by the

\footnotetext{
${ }^{15}$ In the present approach, the default "expected $\mathrm{Cb}^{\prime}$ is the (matrix) SUBJECT referent, and the $\mathrm{Cb}$ is established in the next utterance with a (matrix) (SUBJECT) pronoun, if there is one. More factors such as TOPIC (wa-marking) and Ident (see below) are also relevant to the centering in Japanese. These are discussed in the longer paper in preparation.
} 
property-sharing, as illustrated in the following examples:

(18) [single pronoun]

1. Carl is talking to Tom in the Lab.

2. Terry was just looking for him. [preference: him=: Carl]

(19) [unambiguous two pronouns]

1. Max is waiting for Susan.

2. She invited hlm to dinner.

(18)-2 has only one pronoun and (19)-2 has two pronouns with different gender. In both cases, the nonSUBJECT pronoun naturally corefers with the previous SUBJECT. The property-sharing constraint becomes relevant only in the case of completely ambiguous multipronouns as in (17). Note that this in turn explains why the property-sharing was first recognized for zero pronominals, which lack gender/number/person distinctions altogether.

Explicitly slgnalled parallellsm. The second relevant type of parallelism effect is found in a discourse sequence with explicit linguistic signals for a parallel structure. Examples follow:

(20) [Contrast this with (18)]

1. Carl is talking to Tom in the Lab.

2. Terry wants to talk to him too. [preference: $h$ im=: Tom]

(21) [from Sidner 1979:179]

1. The green Whitierleaf is most commonly

found near the wild rose.

2. The wild violet is found near it too. $<i t=$ : wild rose $>$

Parallelisms in (20) and (21) are clearly signalled with (i) the same verbal expressions (talk to and be found near) and (ii) the word too. In such cases, a version of the property-sharing scheme would propose the correct specification of the single pronoun as the first choice. Since the pronouns are nonSUBJECT, they should co-specify with the nonSUBJECT in the first utterance, which are Tom and the wild rose, respectively. ${ }^{16}$

Significant here is the fact that (21) was a problem case for Sidner's (1979) focusing-based pronoun interpretation algorithm. She in fact concluded that pronoun interpretation involving structural parallelism was a source for anaphora inherently different from focusing:

"Focussing cannot account for the detection of parallel structure, not only because the computation of such structure is poorly understood, but also because focussing chooses different defaults for co-specification than those required for parallelism."(p.236)

If a property-sharing constraint is invoked in interpreting

\footnotetext{
${ }^{16}$ The property of nonSUBJECT may have to be broken up into subclasses (possibly into each grammatical function) when there are more than one nonSUBJECTs in the first utterance.
}

(21)-2, the "wild rose" (nonSUBJECT) overrides the default expected $\mathrm{Cb}$, the "green Whitierleaf" (SUBJECT), as the first-choice referent for the pronoun it (nonSUBJECT). The major advantage of the present property-sharing constraint is its role in combining the effects of both focusing/centering and structural parallelism.

\section{The speaker identification constraint}

\subsection{Ident}

Although correct in most cases, the Centering Constraint as stated in (9) is systematically violated by a certain group of counterexamples in Japanese. This has to do with what Kuno calls empathy, a grammatical feature especially prominent in Japanese, defined as follows:

(22) Empathy (Kuno \& Kaburagi 1977:628)

Empathy is the speaker's identification, with varying degrees, with a person who participates in the event that he describes in a sentence.

I will call it the speaker identification, or simply, identification. ${ }^{17}$ When the main predicate of an utterance selects one of its arguments for the identification locus (henceforth Ident), the speaker automatically identifies (with varying degrees) with the viewpoint of its referent (usually human). The unmarked Ident is the SUBIECT, but some verbs have nonSUBJECT Ident. For instance, among giving/receiving verbs, ageru 'give' and morau 'receive' have SUBJECT Ident, while kureru 'give' has OBJECT2 Ident, ${ }^{18}$ and for going/coming verbs, iku 'go' has SUBJECT Ident while kuru 'come' has nonSUBJECT Ident. Each Ident feature is carried over in a complex predicate made with one of these verbs as the "higher" predicate (e.g., V-kureru 'give the favor of V-ing' Ident=nonSUBJ).

Counterexamples to the constraint stated in (9) are cases with verbs of nonSUBJECT Ident:

\footnotetext{
17"Identification" is a better term than "empathy" in conveying the lack of speaker's emotional involvement and, moreover, it was used in the original definition of empathy in (22). The basic characterization of this notion is fully credited to Kuno and Kaburagi, however.
}

${ }^{18}$ OBJECT2 is the indirect or second object. 
(23)

1. Masao wa Arabia-go o naratte-iru.

Masao TP-SB Arabic OB is-learning

"Masao is learning Arabic."

2. Aruhi $\Phi$ Arabia-zin no zyosei ni atta one-day SB Arabian of lady to met

"One day [he] met an Arabian lady." <Ident=SUBJ> [Cb $<$ SUBJ $>=$ Masao]

3. $\Phi \Phi$ Iroiro sinsetu-ni site-kureta.

SB O2 variously kindly do-gave

" $\Phi$ gave various kind favors to $\Phi$." <Ident=OBJ2>

[strong preference: The lady gave favors to Masao] <zero-SUBJ=: lady, zero-OBJ2=: Masao>

The preferred reading of (23)-3 shows that the zero-Ident-OBJ2 is preferred over the zero-nonIdent-SUBJ for carrying over the $\mathrm{Cb}$ previously realized with a zero-Ident-SUBJ. In other words, when Ident and SUBJECT are split, Ident overrides SUBJECT as the stronger shared property for the zero pronominals that retain the same $\mathbf{C b}$ across adjacent utterances.

Based on the interpretation of various SUBJ/Ident combinations (see Kameyama 1985 Ch.2 for more details), the constraint is restated as follows: ${ }^{19}$

(24) Centering Constraint [Japanese] (final version) Two zero pronominals that retain the same $\mathrm{Cb}$ in adjacent utterances should share one of the following properties (in descending order of preference): 1) Ident-SUBJECT, 2) Ident alone, 3) SUBJECT alone, 4) nonIdent-nonSUBJECT.

The resulting constraint substantiates the role of the zero pronominal in the context of centering in Japanese discourse. The constraint in English need not incorporate the Ident property, however. According to Kuno \& Kaburagi (ibid.), there is only a handful of verbs with SUBJECT Ident (e.g., marry, meet, run into, hear from, receive from) and only one with nonSUBJECT Ident (come up to), none of which propagate with an operation like the Japanese complex verb formation. Moreover, even using these verbs, the Ident effect on pronoun interpretation is not

\footnotetext{
${ }^{19}$ Implicit here are two weakest properties to be shared: 5) nonIdent alone and 6) nonSUBJECT alone. These were left out because of the scarcity of actual instances in discourse. I found, however, that exactly the same scale of shared properties accounts for the possibility of the intra-sentential zero pronominal binding in Japanese, and that the full scale of six properties is actually needed for it (see Kameyama 1986).
}

at all clear in English. ${ }^{20}$

The lack (or dispensability) of the speaker identification constraint does not mean that English centering is less constrained, because English pronouns are inherently more constrained than Japanese zero pronominals by the presence of grammatical features, gender, number, and person. We can view the Ident feature of Japanese zero pronominals as a way to make up for the lack of gender/number/person information available in overt pronouns. The SUBJECT constraint stated in (16), which is simply a subpart of the constraint in Japanese, thus remains adequate in English.

\subsection{Perception verbs: possible link to Ident}

Perception verbs like see/hear, look/sound, etc. anchor the speaker's perspective just like Japanese Ident verbs. For example:

(25)

1. Dan went to a party yesterday.

2. He saw his high school friend Jim. [Cb<SUBJ >=Dan]

3. He looked awfully pale. [preference: Jim looked pale (to Dan).]

(26)

1. Maria finally got her phone reconnected.

2. She called her sister Bella. [Cb $<$ SUBJ $>=$ Maria]

3. She sounded depressed.

[preference: Bella sounded depressed (to Maria)]

Equivalent sequences in Japanese give rise to the same interpretation, that is, the single pronominal element in the third utterance picks out the previous non- $\mathrm{Cb}$. This exceptional case can be explained if verbs like look and sound are used to describe states perceived from the viewpoint of the individual the speaker currently 'identifies with'. As a consequence, the SUBJECT referent of such a description is typically other than the one currently identified with. By making the previous $\mathrm{Cb}$ "the individual the speaker currently identifies with", the preferred readings of (25)-3 and (26)-3 can be explained. This indicates that the speaker's viewpoint is closely related to the $\mathrm{Cb}$ whether or not there is an Ident-based constraint in

${ }^{20}$ Consider the following example:

1. John is my brother.

2. He met Peter at a conference last weekend. <Ident=SUBJ>

3. He came up to him and shook his hand. <Ident=nonSUBJ>

The third utterance should read "Peter came up to John" if Ident overrides SUBJ. More speakers gave the reverse interpretation, however, showing the preference for the SUBJ-SUBJ coreference. 
the language.

Although there is a close relationship between Ident and these perception reports, the 'grammatical' status of the latter is not very clear. In particular, it is questionable whether the effect of perception verbs should be differentiated from commonsense-based interpretations as in the following example: Sam hit Bill on the head. He hit him back on the chin. It is an area open for more detailed studies in the future.

\section{Conclusions}

Within the framework of the Centering approach to pronoun resolution in discourse, I have proposed an additional constraint for Japanese and English. This property-sharing constraint requires that two pronominal expressions that retain the same $\mathrm{Cb}$ across adjacent utterances share a certain common grammatical property. This property has been identified in two dimensions. One has to do with the grammatical function SUBJECT, and the other has to do with the speaker identification property Ident. The latter is necessary for Japanese discourse where the primary $\mathrm{Cb}$-realizer is the zero pronominal, but not for English discourse where it is the (unstressed) overt pronoun. The resulting constraint complements the original Centering rule, accounting for its apparent violations and providing a solution to the interpretation of multi-pronominal utterances.

Two significant implications of the proposed constraint have been discussed. First, the SUBJECT constraint provides an alternative account of anaphora interpretation that appears to be due to structural parallelism. This reconciliation of centering/focusing and parallelism is a major advantage of this constraint. Second, the speaker identification constraint found in Japanese indicates a close association between centering and the speaker's viewpoint. In particular, it sheds light on what underlies the effect of perception reports on pronoun resolution. These results, by drawing on facts in two very different languages, demonstrate the cross-linguistic applicability of the centering framework in general.

The present property-sharing constraint highlights a grammatical aspect that contributes to local discourse coherence. It will be integrated into the default rules which, by ordering the candidate referents for a pronominal expression, control the pragmatic inferences involved in pronoun resolution.

\section{ACKNOWLEDGEMENTS}

My special thanks go to Barbara Grosz for her guidance and encouragement for the work from which this paper developed. I have also greatly profited from discussions with Aravind Joshi and comments on an earlier version by N. Abe, M. Papalaskari, R. Rubinoff, J. Smudski, and
B. Webber.

\section{REFERENCES}

Joshi, Aravind and Scott Weinstein. (1981) Control of Inference: Role of Some Aspects of Discourse Structure-Centering. In Proceedings of the International Joint Conference on Artificial Intelligence. Vancouver, B.C.: 385-387.

Grosz, Barbara J.; Aravind K. Joshi; and Scott Weinstein. (1983) Providing a Unified Account of Definite Noun Phrases in Discourse. In Proceedings of the 21st Annual Meeting of the ACL. Association of Computational Linguistics, Cambridge, Mass: 44-50.

Grosz, Barbara J.; Aravind K. Joshi; and Scott Weinstein. (in preparation) Towards a Computational Theory of Discourse Interpretation. MS. SRI International AI-Center, Menlo Park, CA.

Grosz, Barbara J. and Candace L. Sidner. (1985) The Structure of Discourse Structure. Report No. CSLI-85-39, Center for the Study of Language and Information, Stanford, Califomia. (To appear in Computational Linguistics 1986.)

Kameyama, Megumi. (1985) Zero Anaphora: The Case of Japanese. Ph.D. dissertation, Stanford University, Stanford, California.

Kameyama, Megumi. (1986) Japanese Zero Pronominal Binding: Where Syntax and Discourse Meet. Paper presented at the Second SDF Workshop in Japanese Syntax. Center for the Study of Language and Information, Stanford, California, March 7-9.

Kuno, Susumu and Etsuko Kaburagi. (1977) Empathy and Syntax. Linguistic Inquiry 8: 627-672.

Sidner, Candace L. (1979) Towards a Computational Theory of Definite Anaphora Comprehension in English Discourse. Technical Report TR-537, MIT AI Lab, Cambridge, Mass.

Sidner, Candace L. (1981) Focusing for Interpretation of Pronouns. American Journal of Computational Linguistics. 7(4): 217-231.

Sidner, Candace L. (1983) Focusing in the Comprehension of Definite Anaphora. In: Michael Brady and Robert C. Berwick, Eds., Computational Models of Discourse. MIT press, Cambridge, Mass. 